\title{
The mnemonic effect of choice
}

\author{
Michelle E. Coverdale ${ }^{1}$ • James S. Nairne ${ }^{1}$
}

Published online: 28 February 2019

(C) The Psychonomic Society, Inc. 2019

\begin{abstract}
Making choices during encoding leads to superior memory compared with having the same choices made for you. Evidence also suggests that chosen items might be more memorable than unchosen alternatives. In prior experiments, an incidental memory advantage was found for chosen over unchosen items when participants chose which one of two words would be more useful to a situation. However, it remains uncertain whether this mnemonic benefit is due to the act of choosing or to a better "fit" of chosen items to the encoding situation (congruity). In the present research, we conducted two experiments to dissociate choice and congruity effects. In both experiments, we manipulated choice and congruity and showed mnemonic benefits for chosen words over unchosen words and for congruent words over incongruent words, but these effects did not interact. There is apparently a unique mnemonic benefit for chosen words that cannot be explained by their "fit" to the encoding task.
\end{abstract}

Making choices during a learning episode improves memory. For example, when participants choose to-be-remembered words at encoding, they perform better than a yoked control group who is simply assigned the same words to remember (e.g., Perlmuter, Monty, \& Kimble, 1971; Watanabe \& Soraci, 2004). This "self-choice effect" may be related to self-directed learning effects, in which participants exhibit better memory for information learned while controlling aspects of the learning episode, such as presentation order or duration (Markant, DuBrow, Davachi, \& Gureckis, 2014; Murty, Dubrow, \& Davachi, 2015, 2018; Voss, Gonsalves, Federmeier, Tranel, $\&$ Cohen, 2011). Markant et al. (2014) found that participants who were allowed to control some aspect of the study episode had better memory for the stimuli than did their yoked partners. A mnemonic benefit was associated with making a choice, even if that choice was merely choosing when the stimulus would be presented.

In addition to the overall benefit of choice, people might also preferentially remember chosen over unchosen items. Whenever a choice is made, it involves the selection of one alternative over others. For example, a student may choose which key terms from a study guide to memorize for an up-

Michelle E. Coverdale

mcoverda@purdue.edu

1 Department of Psychological Sciences, Purdue University, 703 Third Street, West Lafayette, IN 47907-2081, USA coming test, or a consumer might choose which one of several brands to purchase when buying a product. Self-choice experiments typically have participants choose to-be-remembered items from a set of alternatives, but rarely compare memory for chosen items to unchosen alternatives. This is because selfchoice experiments typically employ intentional memory tasks. If participants do not believe that they will need to remember the alternatives, then the comparison between chosen and not-chosen items is confounded by factors such as motivation, expectation, and item selection.

However, recent evidence suggests that chosen items might be more memorable than unchosen alternatives, even in incidental memory tasks (Coverdale, Pandeirada, \& Nairne, in press; Cunningham, Brady-Van den Bos, \& Turk, 2011). Across two experiments Coverdale et al. (in press) observed a recall benefit for words chosen in an incidental memory task. Participants were asked to choose which of two objects would be more useful to either a survival scenario or a control scenario. In a surprise freerecall test, both a survival processing benefit (Nairne, Thompson, \& Pandeirada, 2007) and a benefit for chosen items were observed; words that were chosen as more useful were better remembered than the words that were not chosen. The chosen-item benefit did not depend on whether the choice was made relative to the survival or the control scenario.

However, a retention advantage for chosen items does not necessarily mean the act of choosing is responsible for the benefit. In the Coverdale et al. (in press) experiments, when participants chose the word that was more useful to the situation, presumably they picked the word that best fit the assigned scenario. Craik and Tulving (1975) found that when 
stimuli form an integrated unit with the encoding context or question (congruent), they are better recalled than when they do not (incongruent), presumably because participants can use the encoding context as a retrieval cue to help gain access to the target item. This effect, originally demonstrated by Schulman (1974), is known as the congruity effect or congruity principle: An item is more memorable if it is semantically compatible with the encoding context. If participants are instructed to choose the word that is more useful in the situation, they should choose words that are more congruent with the situation. Therefore, a benefit for chosen over unchosen items could merely be an effect of congruity - the chosen words were more congruent with the encoding context and thus more memorable.

In the present research, we disentangle the effect of choice from the effect of contextual congruity. Our goal was to determine if choice, rather than congruity, explains some or all of the observed advantage for chosen items. Across two experiments, we manipulated congruity and choice by having participants choose the more congruent word on half of the encoding blocks and the less congruent word on the other half of the blocks. This manipulation enabled us to compare chosen with unchosen words when those words were either equally congruent or incongruent with the encoding context.

\section{Experiment 1}

Experiment 1 asks whether choice (an item's status as chosen or unchosen), the item's congruity with the scenario, or a combination of both lead to improved memory for an item. To separate these two factors (choice and congruity), we instructed participants in one condition to choose which of two objects would be more useful in a survival situation (congruent) and participants in another condition to choose which object would be less useful (incongruent). With this design, we were able to look at the mnemonic effect of choice (chosen vs. not chosen) at two levels of matched item-scenario congruity (congruent vs. incongruent).

\section{Method}

Participants Forty-eight native-English-speaking Purdue University undergraduates (25 female) were recruited from an introductory psychology class and participated for partial course credit.

Materials Twenty-four two-object pairs (e.g., whistle and envelope) were used. All 48 words were objects with familiarity, concreteness, and imageability values above 450 , based on the Medical Research Council Psycholinguistic Database (Coltheart, 1981). All participants saw the same pairs of words.
Word pairs were divided into four blocks of six pairs. In alternating blocks, participants made a decision about which word was more useful (M) or less useful (L) to a survival situation. Decision type (more/less) alternated between blocks and was counterbalanced across participants (i.e., half of the participants did MLML and half did LMLM). Word pairs within each block were held constant across participants, such that each person saw the same set of six pairs in the first block regardless of the decision type. Within each block, presentation order of the pairs was randomized. Except for the counterbalancing of the decision type across the block and ordering of the pairs within each block, all other variables were held constant across participants.

Procedure Participants were run in groups of one to four, on individual computers, in sessions lasting fewer than 30 minutes. Instructions were presented before each task and before each block of choices. For both decision types (more/less), participants made their choices about the same survival situation. The survival situation and instructions for the decisions types were as follows:

Survival situation. "We would like you to imagine that you are stranded in the grasslands of a foreign land, without any basic survival materials. Over the next few months, you'll need to find steady supplies of food and water and protect yourself from predators."

More useful. "We are going to show you pairs of words, representing objects, and we would like you to decide which of the two objects would be MORE useful to you in the survival situation."

Less useful. "We are going to show you pairs of words, representing objects, and we would like you to decide which of the two objects would be LESS useful to you in the survival situation."

Before the first block of both decision tasks, participants performed two practice trials that were excluded from all analyses. Each pair of objects was accompanied by the prompt "Which is MORE (or LESS) useful in the survival situation?" Participants had 5 seconds per pair to decide. Participants made their choices by clicking, with the mouse, on the word that they wished to select. In total, participants made 24 decisions, 12 for each decision type.

Following the last block of choice trials there was a 2minute distractor task. During this task, participants saw a single-digit number on the screen for 2 seconds and made a parity judgment by clicking on either the word "even" or "odd." Following the distractor task, participants were given a 5-minute surprise free-recall task. Participants were asked to recall the names of all the objects that were encountered during the choice task, regardless of what choice they had made. Participants typed the names of the recalled objects into an onscreen text box. 


\section{Results and discussion}

For all analyses, we set alpha $=.05$ (two-tailed). For the purpose of these comparisons we considered words that were chosen in the "more useful" condition and not chosen in the "less useful" condition to be congruent with the scenario. Words that were not chosen in the "more useful" condition or were chosen in the "less useful" condition were considered to be incongruent with the scenario. Therefore, we had four categories of words: chosen, congruent; not chosen, congruent; chosen, incongruent; not chosen, incongruent (see Fig. 1).

The results of main interest are shown in Fig. 2. The choice effect (chosen vs. unchosen) is plotted separately for the matched congruent and incongruent items. Using a repeatedmeasures analysis of variance (ANOVA), we found a main effect of choice, $F(1,47)=10.35, p=.002, M S E=.02, \eta_{\mathrm{p}}^{2}$ $=.18$, and a main effect of congruity, $F(1,47)=25.41, p<$ $.001, M S E=.03, \eta_{\mathrm{p}}{ }^{2}=.35$. Participants had better memory for words that they had selected and better memory for words that were relatively more useful to survival. We found no interaction between choice and congruity, $F(1,47)<1$. Because null hypothesis testing only allows one to calculate the probably of obtaining the data given a null effect, we conducted an additional Bayesian analysis to calculate the likelihood of a null interaction between choice and congruity given our data. Using Masson's (2011) guidelines, we calculated the posterior probabilities of the null $\left(\mathrm{H}_{0}\right)$ and the alternative $\left(\mathrm{H}_{1}\right)$ hypotheses given our data (D). The posterior probabilities were $P_{\mathrm{BIC}}\left(\mathrm{H}_{0} \mid \mathrm{D}\right)=.833$ and $P_{\mathrm{BIC}}\left(\mathrm{H}_{1} \mid \mathrm{D}\right)=.167$, indicating positive evidence in favor of a null interaction.

If differential congruity is responsible for the chosen-item benefit, then there should have been a main effect of congruity, but no main effect of choice. Specifically, congruent items (chosen in the more condition and not chosen in the less condition) should have been more memorable than incongruent items (not chosen in the more condition and chosen in the less condition), but choosing, by itself, should not confer a benefit. This is because our design essentially equates the amount of congruity across chosen and unchosen items. Still, a strong choice effect emerged for both the matched congruent and incongruent items. Moreover, the fact that congruity and

\section{Decision Type

\begin{tabular}{lll|} 
& \multicolumn{1}{c}{ More } & \multicolumn{1}{c}{ Less } \\
Congruent & whistle & whistle \\
Incongruent & envelope & envelope \\
& &
\end{tabular}

Fig. 1 For each pair of words (e.g., whistle and envelope), one word was chosen (black) and one was not chosen (white). Based on the type of decision that the participant was instructed to make the chosen word was either the congruent word (whistle) or the incongruent word (envelope) choice failed to interact suggests that different mechanisms probably mediate the two effects. Researchers commonly assume that congruity effects arise because words that fit the encoding context have a greater potential for elaboration; for example, Craik and Tulving (1975) showed that deeper levels of processing, along with sentence complexity, produce larger congruity effects because the potential for elaboration is greater (see also Fisher \& Craik, 1977). If the choice effect is produced by a similar mechanism, item-context elaboration, then a larger choice effect should have been found for congruent items, where the potential for elaboration is greater. Our finding that congruity and choice both benefit recall but do not interact suggests that the memory benefit from choosing an item is independent of the item's congruity to the encoding context.

\section{Experiment 2}

In Experiment 1 we found a mnemonic benefit for chosen items that was independent of congruity between the item and the encoding situation. However, it is conceivable that we failed to equate the amount of item-scenario congruity adequately between conditions. Because the usefulness decision in Experiment 1 was subjective, different items could have been sampled across the chosen and unchosen conditions - for example, the items that were chosen in the "more" condition could have been different from the items that were not chosen in the "less" condition. To check on this possibility, we correlated the proportion of times one word in a pair was chosen by participants in the "more" task with the proportion of times the same word was not chosen by participants in the "less" task. We found that there was a strong positive correlation $(r=.92, p<.001)$, which suggests that the perception of an object's usefulness was consistent across participants.

Still, in Experiment 2 we changed the choice task from one requiring a subjective decision to one requiring an objective decision, about which word is more representative of a category. As before, participants saw pairs of words and selected one of the words based on a prompt. Each pair of words was accompanied by a category label that applied to only one word. For example, a participant might see the words golf and boot accompanied by the category "a sport," and they would need to choose the word that is either more representative of the category (golf) or less representative of the category (boot). Crucially, we manipulated the category prompt between subjects such that half of the participants saw prompts that were congruent with one set of words whereas the other half of participants saw prompts that were congruent with the other set of words. In this way, not only did each word participate as both a chosen and an unchosen item across 

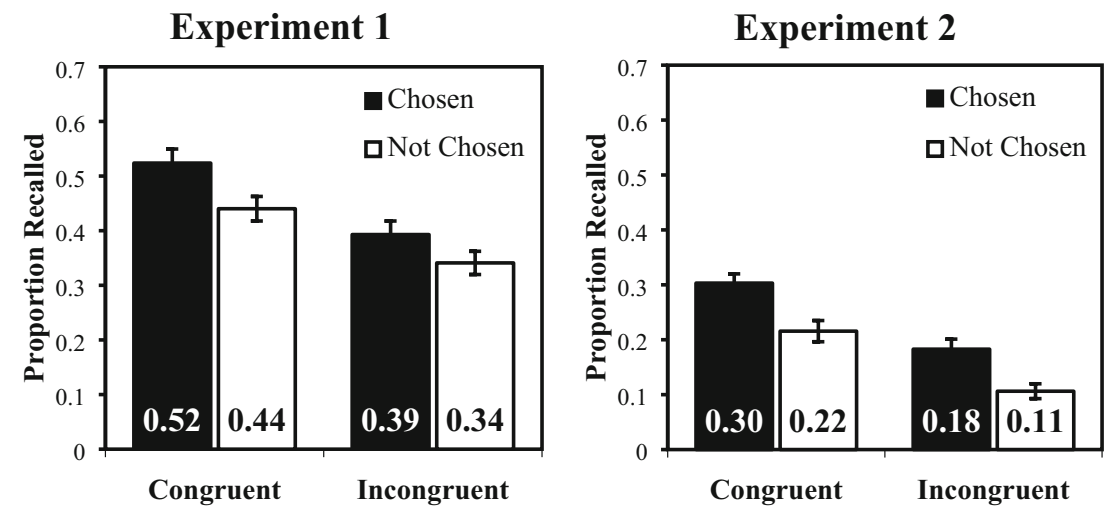

Fig. 2 Free recall performance by choice and congruity in Experiment 1 and Experiment 2. Error bars represent the standard error of the mean

participants, each word also participated as both a congruent and an incongruent item across participants (see Fig. 3).

We also changed the way participants physically responded in the choice task. In Experiment 1 participants used the mouse to choose words; consequently, they may have spent more time looking at the chosen word (because they are coordinating their mouse movement in that direction); hence, differential attention to the chosen word might have influenced the observed mnemonic benefit. In Experiment 2, we altered the choice task such that participants clicked on either the word left or right to make their selection instead of the on the chosen word itself.

\section{Method}

Participants We performed a statistical power analysis to estimate sample size for this experiment based on the choice effect found in Experiment 1. Using G*Power 3.1 (Faul, Erdfelder, Buchner, \& Lang, 2009) the estimated sample size with alpha $=.05$ and power $=.95$ was $N=64 .{ }^{1}$ Sixty-four Purdue University undergraduates (39 females) participated in exchange for partial course credit.

Materials Forty word and category stimuli were selected from the Van Overschelde, Rawson, and Dunlosky (2004) category norms. Twenty word pairs were presented in blocks of five. Each word pair was associated with two category descriptions, one congruent and one incongruent for each word. Half of the descriptions were presented in one version (A) and the other half were presented in the other version (B) such that each word was congruent in one version and incongruent in the other. Version (A or B) and decision task order (MLML or LMLM) were counterbalanced between subjects, resulting in four counterbalanced versions ( $n=16$ per version).

The length of the category description (in number of characters) was matched between the two versions (A/B). The set of

\footnotetext{
${ }^{1}$ For the power analysis we used the partial eta squared reported in Experiment 1 that was output by SPSS.
}

congruent words in the two versions were matched in average word length, familiarity, concreteness, and imageability (Coltheart, 1981). Importantly, the words in the two versions were also matched for their category representativeness, as measured by the proportion of participants in Van Overschelde et al. (2004), who generated the target word when cued with the category. All other materials were the same as Experiment 1.

Procedure The general procedure was the same as in Experiment 1, except that participants were instructed to make decisions based on which word was representative of a category and to click on the word left or right to indicate which word they wanted to select.

For each pair of words, the prompt "Which is MORE (or LESS) representative of the category?" appeared at the top of the screen, and below the prompt was the category. Under the category were the two target words, and below the two target words were the words "left and right". Participants were given 7 seconds to read the category and words and make their decision. In total, participants made 20 decisions, 10 for each decision type (more/less representative). The distractor task and free-recall task were as described in Experiment 1.

\section{Results and discussion}

The results of interest are shown in Fig. 2. There was a significant reduction in overall recall levels between Experiments 1 and 2. This is likely due to the nature of the encoding task; in Experiment 1, participants were engaging in survival processing, which is an extremely robust form of encoding (e.g., Nairne et al., 2007). But there are additional differences between the experiments, such as the target stimuli and the ease of the choice decision, that might also be affecting the final recall level. We used a repeated-measures ANOVA and replicated the effects reported in Experiment 1. We found a main effect of choice, $F(1,63)=36.09, p<.001, M S E=.01, \eta_{\mathrm{p}}{ }^{2}=$ .36 , and a main effect of congruity, $F(1,63)=74.02, p<.001$, $M S E=.01, \eta_{\mathrm{p}}{ }^{2}=.54$, but no interaction between choice and congruity, $F(1,63)<1$. We again conducted a Bayesian 


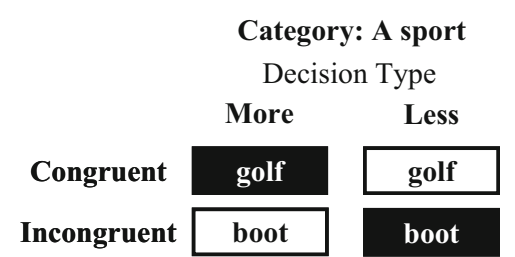

Fig. 3 Across participants, each word appeared in all four conditions(e.g., when golf was presented with the category "a sport," it was congruent because it is a member of the category, and it was either chosen (black) on not chosen (white) based on whether the participant was instructed to

analysis on the interaction and found posterior probabilities of $P_{\mathrm{BIC}}\left(\mathrm{H}_{0} \mid \mathrm{D}\right)=.889$ and $P_{\mathrm{BIC}}\left(\mathrm{H}_{1} \mid \mathrm{D}\right)=.111$. This is interpreted as positive evidence in favor of a null interaction. In sum, words that were a member of the presented category were more memorable those that were not and, as in Experiment 1 , words that were chosen were more memorable than those that were not chosen. The effect of choice on recall did not change based on whether the word was congruent or incongruent with the category.

An important feature of this experiment is that we manipulated both the congruity between the word and the encoding context (the category) and level of choice (chosen/not chosen) for each word. Participants were highly accurate when making their choice. The correct response was made $99 \%$ of the time $(S D=.03)$. This means that when we compare across conditions, we are essentially comparing recall of the same words under four different conditions. That is, choosing a word (e.g., golf) as representative of its category (e.g., a sport) led to better memory than when the same word was presented with the same category label but it was not chosen because the instructions were to choose the less representative word; likewise, if the word was presented with an incongruent category (e.g., a type of footwear), then it was more memorable if it was chosen as being "less representative" of the category than if it was not chosen as being "more representative" of the category. This experiment provides additional evidence that there is something other than congruity that improves memory for chosen items.

\section{General discussion}

We designed two experiments to dissociate the mnemonic benefit for chosen items from the effect of item-context congruity. Participants chose which word of a pair was more or less useful to survival (Experiment 1) or more or less representative of a category (Experiment 2). We found a mnemonic advantage for chosen words in both experiments. We also found a mnemonic advantage for congruent items, but this effect did not interact with the effect of choice; that is, the size of the choice effect did not depend on the level of congruity between the word and the encoding context. These results

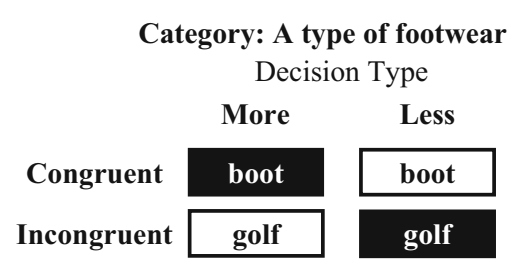

choose the more representative or less representative word). When the word golf was presented with the category "a type of footwear," it was incongruent, and it was either chosen or not chosen based on the type of decision the participant was instructed to make (more/less)

suggest that something other than congruity is responsible for the chosen-item mnemonic benefit.

Although a mnemonic benefit for chosen items has been seen in previous experiments (e.g., Coverdale et al., in press), the effect of choice has been confounded by other variables. Here, we replicated the chosen-item effect while controlling for congruity between the word and the encoding situation. In fact, the effects of choice and congruity appeared to be independent, suggesting that different mechanisms mediate the two effects. We also found that the chosen-item benefit did not depend on participants interacting with the word while making their selections (i.e., clicking on the chosen word using the mouse).

These findings importantly extend research on the mnemonic effects of choosing. Prior research has shown mnemonic benefits for choice conditions over no choice controls (e.g., Markant et al., 2014; Murty et al., 2015, 2018), but there has not been a systematic investigation comparing recall for chosen items and unchosen alternatives within a choice condition. The present experiments provide evidence that within a choice condition there is a memory benefit for the items that are selected compared with the unselected alternatives. This finding helps to constrain prior theoretical accounts of choice benefits. For example, previous researchers have suggested that the mnemonic benefits associated with choosing might arise because participants feel an increased sense of agency when they are allowed to make choices. In the present experiments participants made choices on every trial, so "perceived agency" cannot account for the mnemonic benefits obtained here.

Moreover, as discussed above, by dissociating choice and congruity we have provided evidence suggesting that congruity and choice are independent effects. The two effects did not interact, reducing the chances that they are caused by the same mechanism. Congruity effects are commonly explained through differential elaboration (Craik \& Tulving, 1975). Congruent items fit the encoding context, so the encoded trace is richer or more elaborate, which, in turn, increases the availability of diagnostic retrieval cues. As a case in point, in Experiment 2, participants could use the category name as a retrieval cue for accessing a congruent item, but not an incongruent one. The act of choosing an item might lead to enhanced elaboration as well, but then one would reasonably expect congruity and choice to interact. The congruity effect 
commonly interacts with depth of processing because shallow items offer fewer opportunities for elaboration than do congruent items (see Craik \& Tulving, 1975). Similarly, if elaboration is the operative mechanism, then there should have been a smaller choice effect for the incongruent items because the potential for elaboration is lower.

It is possible that the choice benefit is related to the mere ownership effect (e.g., Beggan, 1992). When someone chooses something, even if they are choosing it as being less useful in a given context, they may feel a sense of ownership over the choice, which might then lead to better memory for the chosen item. An alternative explanation is that perceived value drives the mnemonic benefit for chosen items. It has been suggested that humans selectively remember information that is important or relevant while forgetting information that is not (Stefanidi, Ellis, \& Brewer, 2018). Participants have better memory for items that are assigned a higher value at encoding (Castel, Farb, \& Craik, 2007; Stefanidi et al., 2018), an effect known as value-directed remembering. The act of choosing may be a signal that the chosen item is important. We typically choose things for a reason, presumably because they are of some value or importance, so we may have a bias to assume that chosen things are valuable. If subjects perceive chosen items as being more important/valuable, then valuedirected remembering could account for the mnemonic benefit of chosen items over unchosen items.

Additionally, if we usually choose things because they are valuable or relevant, then asking people to choose the less useful/representative item might have caused them to experience cognitive dissonance, an internal tension (Festinger \& Carlsmith, 1959), due to the mismatch between their own beliefs (that the item is less useful/representative) and their action of choosing the item. In this case, participants might change their attitudes about the chosen items and perceive them as more useful to reduce the tension from the inconsistency between the choice they made and how they feel about the item. This is similar to the idea of choice-induced preference change, wherein choosing one item from a pair of equally preferred items decreased preference for the unchosen item due to the dissonance from rejecting an item that is liked (e.g., Izuma et al., 2010).

In summary, two experiments showed a mnemonic benefit for chosen items independent of congruity effects - that is, participants have better memory for chosen items and for congruent items, but the effect of choice does not depend on whether items are congruent or incongruent. This suggests that the chosen-item effect is caused by something other than the congruity of the items to the encoding situation. These experiments will hopefully lay a foundation for future research investigating the chosen-item effect. Choices are an integral part of our lives; we choose what to eat, which products to consume, and how to delegate our time (to name only a few examples); therefore, it is important that we explore and improve our understanding of the effects of choice on memory.
Acknowledgements This research was supported, in part, by a grant from the National Science Foundation (BCS-1532345). The authors wish to thank Josefa Pandeirada for comments regarding this research.

Publisher's note Springer Nature remains neutral with regard to jurisdictional claims in published maps and institutional affiliations.

\section{References}

Beggan, J. K. (1992). On the social nature of nonsocial perception: The mere ownership effect. Journal of Personality and Social Psychology, 62(2), 229-237.

Castel, A. D., Farb, N. A., \& Craik, F. I. (2007). Memory for general and specific value information in younger and older adults: Measuring the limits of strategic control. Memory \& Cognition, 35(4), 689700 .

Coltheart, M. (1981). The MRC psycholinguistic database. The Quarterly Journal of Experimental Psychology, 33, 497-505.

Coverdale, M. E., Pandeirada, J. N. S., \& Nairne, J. S. (in press). Survival processing in a novel choice procedure. American Journal of Psychology.

Craik, F. I., \& Tulving, E. (1975). Depth of processing and the retention of words in episodic memory. Journal of Experimental Psychology: General, 104(3), 268-294.

Cunningham, S. J., Brady-van den Bos, M., \& Turk, D. J. (2011). Exploring the effects of ownership and choice on self-memory biases. Memory, 19(5), 449-461.

Faul, F., Erdfelder, E., Buchner, A., \& Lang, A.-G. (2009). Statistical power analyses using $\mathrm{G}^{*}$ Power 3.1: Tests for correlation and regression analyses. Behavior Research Methods, 41, 1149-1160.

Festinger, L., \& Carlsmith, J. (1959). Cognitive consequences of forced compliance. Journal of Abnormal and Social Psychology, 58(2), 203-210.

Fisher, R. P., \& Craik, F. I. M. (1977). Interaction between encoding and retrieval operations in cued recall. Journal of Experimental Psychology: Human Learning and Memory, 3, 701-711.

Izuma, K., Matsumoto, M., Murayama, K., Samejima, K., Sadato, N., \& Matsumoto, K. (2010). Neural correlates of cognitive dissonance and choice-induced preference change. Proceedings of the National Academy of Sciences of the Unites States of America, 107(51), 22014-22019. https://doi.org/10.1073/pnas.1011879108

Markant, D., DuBrow, S., Davachi, L., \& Gureckis, T. M. (2014). Deconstructing the effect of self-directed study on episodic memory. Memory \& Cognition, 42(8), 1211-1224.

Masson, M. E. (2011). A tutorial on a practical Bayesian alternative to null-hypothesis significance testing. Behavior research methods, 43(3), 679-690.

Murty, V. P., DuBrow, S., \& Davachi, L. (2015). The simple act of choosing influences declarative memory. Journal of Neuroscience, 35(16), 6255-6264.

Murty, V. P., DuBrow, S., \& Davachi, L. (2018). Decision-making increases episodic memory via postencoding consolidation. Journal of Cognitive Neuroscience. Advanced online publication. https://doi. org/10.1162/jocn_a_01321

Nairne, J. S., Thompson, S. R., \& Pandeirada, J. N. S. (2007). Adaptive memory: Survival processing enhances retention. Journal of Experimental Psychology: Learning, Memory, and Cognition, 33(2), 263-273.

Perlmuter, L. C., Monty, R. A., \& Kimble, G. A. (1971). Effect of choice on paired-associate learning. Journal of Experimental Psychology, 91(1), 47-53.

Schulman, A. I. (1974). Memory for words recently classified. Memory \& Cognition, 2(1), 47-52. 
Stefanidi, A., Ellis, D. M., \& Brewer, G. A. (2018). Free recall dynamics in value-directed remembering. Journal of Memory and Language, $100,18-31$.

Van Overschelde, J. P., Rawson, K. A., \& Dunlosky, J. (2004). Category norms: An updated and expanded version of the Battig and Montague (1969) norms. Journal of Memory and Language, 50(3), 289-335.
Voss, J. L., Gonsalves, B. D., Federmeier, K. D., Tranel, D., \& Cohen, N. J. (2011). Hippocampal brain-network coordination during volitional exploratory behavior enhances learning. Nature Neuroscience, 14(1), 115-120.

Watanabe, T., \& Soraci, S. A. (2004). The self-choice effect from a multiple-cue perspective. Psychonomic Bulletin \& Review, 11(1), $168-172$. 